\title{
Therapeutic Outcome Assessment on Post-Partum Sepsis
}

\author{
Jagatheesh Kaliaperumal1, Neha Sajan Mathews², Pushpa Devi' ${ }^{3}$, Unmesh Santhpur ${ }^{4}$, A. Pandurangan ${ }^{5}$, Amit Mittal6, \\ Md Shamshir Alam ${ }^{7}$
}

\begin{abstract}
1Department of Pharmacy, MM College of Pharmacy, Maharishi Markandeswar (Deemed to be University), Mullana, Ambala, Haryana, India. ${ }^{2}$ Department of Pharmacy, MM College of Pharmacy, Maharishi Markandeswar (Deemed to be University), Mullana, Ambala, Haryana, India. ${ }^{3}$ Department of Pharmacy, MM College of Pharmacy, Maharishi Markandeswar (Deemed to be University), Mullana, Ambala, Haryana, India. ${ }^{4}$ Department of Pharmacy, Maharishi Markandeswar Institute of Medical Sciences and Research Maharishi Markandeswar (Deemed to be University), Mullana, Ambala, Haryana, India. ${ }^{5}$ Department of Pharmacy, MM College of Pharmacy, Maharishi Markandeswar (Deemed to be University), Mullana, Ambala, Haryana, India. ${ }^{6}$ Department of Pharmacy, Maharishi Markandeswar Institute of Medical

Sciences and Research Maharishi Markandeswar (Deemed to be University), Mullana, Ambala, Haryana, India.

7Department of Pharmacy, MM College of Pharmacy, Maharishi Markandeswar (Deemed to be University), Mullana, Ambala, Haryana, India.
\end{abstract}

\section{ABSTRACT}

\section{BACKGROUND}

Postpartum sepsis or puerperal sepsis, an infectious condition which develops within six weeks of after delivery, is one of the underlying causes that contribute to maternal death, and its associated complications, not only in developing countries, but also in developed countries. The strong prevalence of postpartum sepsis is mainly due to the literacy rate of the country. The study was carried out to throw light on the aetiology and management of postpartum sepsis in obstetrics \& gynaecology department at MM Institute of Medical Science and Research and Hospital, Maharishi Markandeshwar (Deemed to be University), Haryana.

\section{METHODS}

Data was obtained through case records of the individual participants. The aetiological organism that invaded the participants was found through microbial culture test from patient samples. Antibiogram was done to determine the sensitivity of the organism to various drugs in the particular participant.

\section{RESULTS}

Out of the 25 samples, the most commonly isolated organism was Enterococcus followed by Klebsiella species. These micro-organisms were found to be resistant to commonly used antibiotics such as Streptomycin, Vancomycin, Linezolid, Nitrofurantoin, Tetracycline and Tobramycin.

\section{CONCLUSIONS}

Early identification and diagnosis can play a key role in reducing morbidity and mortality in maternal sepsis. Without adequately paraphernalia the burden of maternal infections during pregnancy, it would become challenging to improve the health status of neonate.

\section{KEY WORDS}

Enterococcus and Klebsiella species are the common bacteria isolated in postpartum sepsis. Enterococcus species was found to be highly susceptible to streptomycin, vancomycin and linezolid and Klebsiella species to tetracycline, tobramycin and chloramphenicol. Drugs to which organisms were found to be resistant in the study were levofloxacin, penicillin, norfloxacin, ciprofloxacin, ceftriaxone, erythromycin, cefepime and cephalothin. Thus, these drugs may not be prescribed in the empirical therapy of postpartum sepsis. To counteract the burden of puerperal infections on neonatal mortality, minimising infection in the peri-partum period using appropriate measures and antibiotics prophylaxis on one side, and reducing the risk of maternal infection through antenatal care and nutritional supplementation are needed.
Corresponding Author: Dr. Jagatheesh Kaliaperumal, Professor, Department of Pharmacy, MM College of Pharmacy, Maharishi Markandeswar (Deemed to be University), Mullana, Ambala, Haryana, India. E-mail: kjagatheesh@gmail.com

\section{DOI: $10.14260 /$ jemds/2019/805}

Financial or Other Competing Interests: None.

How to Cite This Article: Kaliaperumal J, Mathews NS, Devi P, et al Therapeutic outcome assessment on postpartum sepsis. J. Evolution Med. Dent. Sci. 2019;8(49):3720-3723, $10.14260 /$ jemds $/ 2019 / 805$

Submission 10-10-2019, Peer Review 23-11-2019, Acceptance 29-11-2019, Published 09-12-2019. 


\section{BACKGROUND}

Motherhood is a very beautiful phase a woman encounters in her life. It is a state of happiness, joy and a time of nurturing a baby but sometimes it can turn in to a difficult phase after delivery. According to World Health Organization (WHO) postpartum sepsis is infection of the genital tract occurring at any time between the rupture of membranes or labour within $42^{\text {nd }}$ day of postpartum which comprises of clinically significant pain in pelvic region or vaginal discharge with pus cells and smell with elevated body temperature.1,2 Postpartum sepsis results a significant mortality and morbidity worldwide. As we look keen on global indicators The International Conference on Population and Development (1994) had recommended reduction in maternal mortality by at least 50 per cent of the 1990 levels by the year 2000 and further one half by the year $2015 .{ }^{3}$ The Millennium Development Goals (MDG) has set the target of achieving 200 maternal deaths per lakh of live births by 2007 and 109 per lakh of live births by 2015.4 The incidence of maternal mortality rate in India is 408 per $1,00,000$ live births and $13 \%$ of maternal mortality is due to infection. The maternal mortality rate is higher in Odessa as compare to Kerala which is low around $[87 / 100,000] .{ }^{5}$ In India, various studies were conducted in the states like in Haryana and Maharashtra. In Haryana Seventy-four percent showed at least one morbidity and there were 1.75 reported morbidities per woman per postpartum period. ${ }^{6}$ In Maharashtra (43\%) reported postpartum morbidity and it is associated with adverse outcome. ${ }^{7}$

The prevalence of maternal mortality ratio gets affected by various socio economic factors such as lack of education, status of women, economic dependency, prolonged socio economic status, poor balance diet, lack of hygiene, lack of access to various services and sometime usually gender partiality. ${ }^{5}$ Obstetric haemorrhage is the world's leading cause of maternal mortality, responsible for an estimated 127 000 deaths annually. Postpartum haemorrhage (PPH) is the most common type of obstetric haemorrhage and report for the majority of the 14 million cases that occur each year. Failure of the uterus to contract adequately after childbirth is the most common cause of postpartum haemorrhage. In the absence of timely and appropriate action, a woman could die within a few hours.

Puerperal pyrexia and sepsis are the leading causes of preventable maternal morbidity and mortality not only in developing countries but also have in developed countries. ${ }^{8}$ Thus failure to identify the seriousness of an infection by pregnant women, family members and health care providers is a major universal risk factor. ${ }^{9}$ Hence, there is an urgent need to assess the therapeutic outcome pertaining to postpartum sepsis, this prospective study describes the antibiotic therapy outcome in post-partum sepsis case in tertiary care teaching hospital. The need of present study is to reduce morbidity \& mortality due to postpartum sepsis and to re-evaluate the existing guidelines for management postpartum sepsis. The improvement of post-partum health status is need of the hour this area is need to be urgently reinforced in country like India the fertility rate of women goes beyond 2.5 in few states.

\section{METHODS}

This prospective observational study was done in obstetrics \& gynaecology department at Maharishi Markandeshwar deemed to be University medical College \& Hospital. This study was undertaken in the duration for 6 months. The patients were recruited from gynaecology and obstetrics wards after being diagnosed as postpartum sepsis by the physician. A total of 25 postpartum sepsis participants were included in this study. Information regarding culture and antibiogram test for postpartum sepsis was obtained from microbiology lab. The project work was initiated in accordance with the Principle of standard clinical guidelines. The patient consent was taken for the participation of the volunteers. The confidentiality of patient data was maintained throughout the study period.

\section{Statistical Analysis}

All the information about the medications, culture reports and antibiogram test reports were documented in excel sheet for calculation and evaluation of data and the data was further treated with PRISM8 GraphPad for statistical analysis.

\section{RESULTS}

During 6 months of study 25 patients were clinically diagnosed for post-partum sepsis. Different age and parity of women were admitted but the highest admission was at the age of $20-25$ years. $54 \%$ of patients had to undergo caesarean section. In the present study the most isolated organism was found to be Enterococcus species followed by the Klebsiella which was distinct from the other study done on postpartum sepsis. The existing guideline and the current therapy show that a combination of antibiotics is given Ampicillin 2 gram IV every 6 hours, Gentamicin $5 \mathrm{mg} / \mathrm{kg}$ body weight IV every 24 hours and Metronidazole $500 \mathrm{mg}$ IV every 8 hours, give IV fluid and check the vital sign regularly.10,11 The result of the analysed data was presented in figures, tables and pie chart according to the data collected.

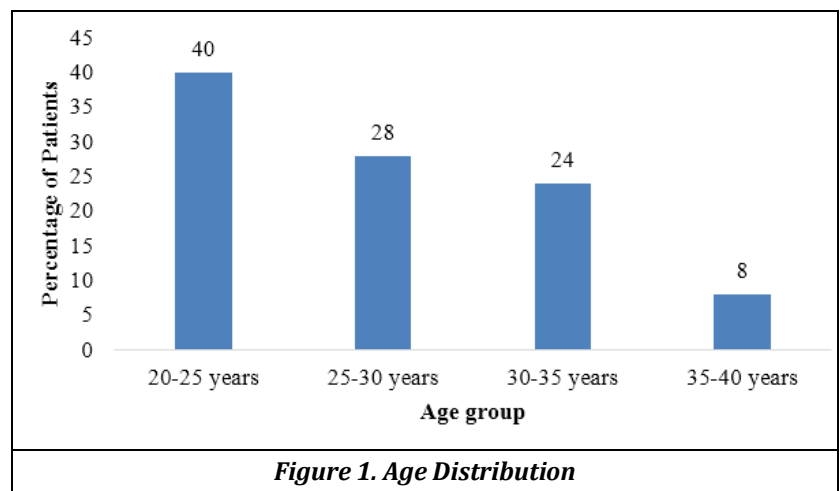



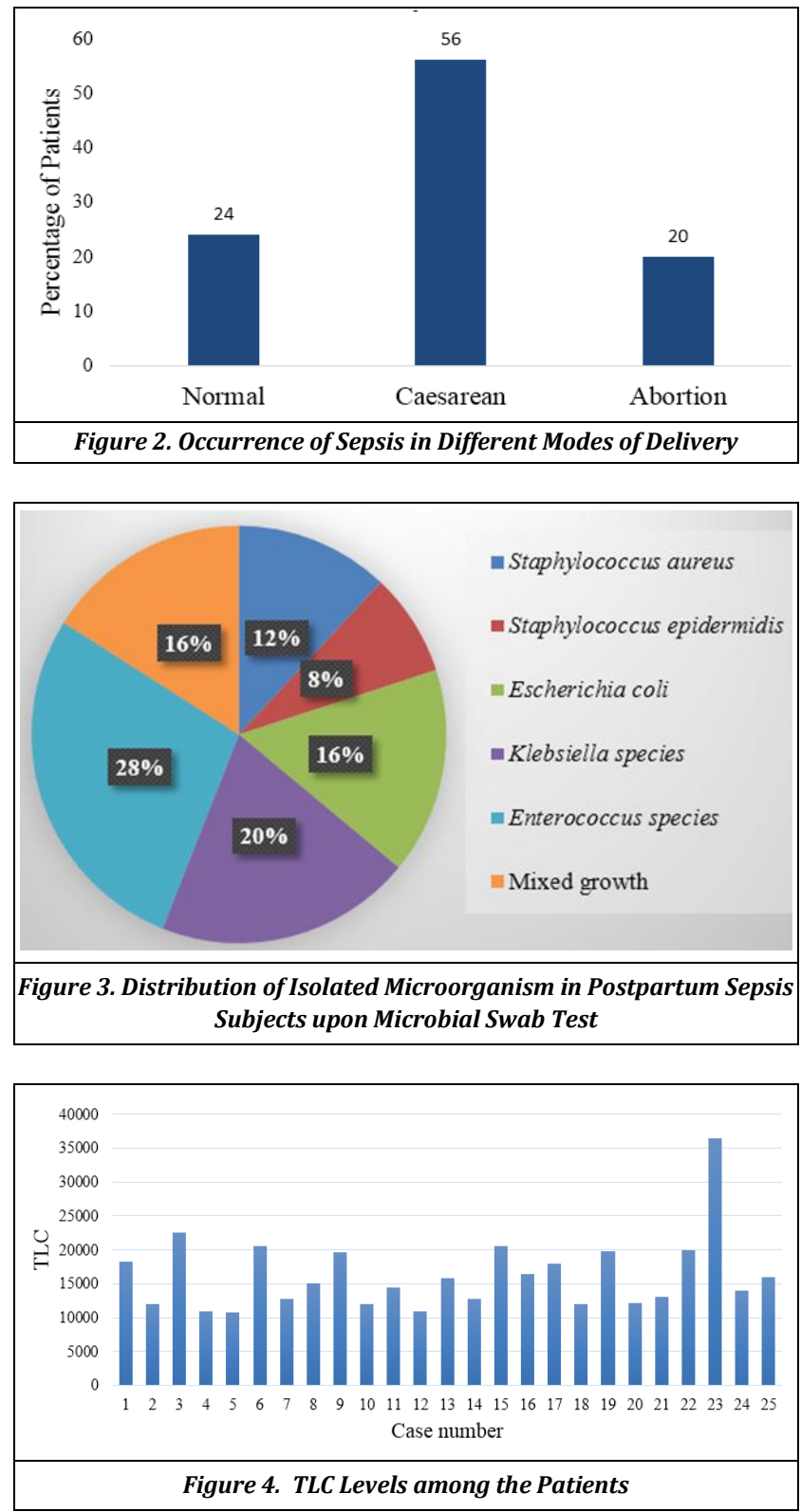

\begin{tabular}{|c|c|c|c|}
\hline Antibiotics & Sensitivity (\%) & Resistance (\%) & Total \\
\hline Co-trimoxazole & 26.67 & 73.33 & 60 \\
\hline Imipenem & 88.89 & 11.11 & 36 \\
\hline Meropenem & 84.61 & 15.39 & 52 \\
\hline Tetracycline & 45 & 55 & 80 \\
\hline Levofloxacin & 0 & 100 & 88 \\
\hline Tobramycin & 66.67 & 33.33 & 48 \\
\hline Ampicillin & 30 & 70 & 80 \\
\hline Piperacillin/ Tazobactam & 57.14 & 42.86 & 56 \\
\hline Amoxicillin/Clavulanic acid & 42.86 & 57.14 & 56 \\
\hline Chloramphenicol & 80 & 20 & 60 \\
\hline Gentamycin & 56.52 & 43.48 & 92 \\
\hline Cefotaxime & 0 & 100 & 36 \\
\hline Penicillin & 0 & 100 & 64 \\
\hline Streptomycin & 100 & 0 & 44 \\
\hline Vancomycin & 100 & 0 & 64 \\
\hline Norfloxacin & 0 & 100 & 44 \\
\hline Linezolid & 100 & 0 & 64 \\
\hline Ciprofloxacin & 0 & 100 & 92 \\
\hline Ceftriaxone & 0 & 100 & 52 \\
\hline Azithromycin & 33.33 & 66.67 & 12 \\
\hline Ofloxacin & 40 & 60 & 20 \\
\hline Erythromycin & 0 & 100 & 20 \\
\hline Clindamycin & 16.67 & 83.33 & 24 \\
\hline Cefoxitin & 0 & 100 & 20 \\
\hline Cefepime & 0 & 100 & 20 \\
\hline Cephalothin & 0 & 100 & 20 \\
\hline Nitrofurantoin & 100 & 0 & 28 \\
\hline Fosfomycin & 100 & 0 & 24 \\
\hline
\end{tabular}

\begin{tabular}{|c|c|c|c|}
\hline Antibiotic Therapy & Percentage & Sensitivity & Resistance \\
\hline Meropenem & 12 & 84.61 & 15.39 \\
\hline Linezolid & 36 & 100 & 0 \\
\hline Clindamycin & 12 & 16.67 & 83.33 \\
\hline Metronidazole & 60 & NA & NA \\
\hline Erythromycin & 4 & 0 & 100 \\
\hline Amoxicillin/Clavulanic acid & 24 & 42.86 & 57.14 \\
\hline Ceftriaxone & 44 & 0 & 100 \\
\hline Azithromycin & 8 & 33.33 & 66.67 \\
\hline Gentamycin & 64 & 56.52 & 43.48 \\
\hline Tobramycin & 4 & 66.67 & 33.33 \\
\hline Doxycycline & 4 & NA & $\mathrm{NA}$ \\
\hline Amikacin & 4 & NA & NA \\
\hline Ampicillin & 4 & 30 & 70 \\
\hline Piperacillin/ Tazobactam & 12 & 57.14 & 42.86 \\
\hline Norfloxacin & 4 & 0 & 100 \\
\hline Faropenem & 4 & NA & NA \\
\hline Ciprofloxacin & 4 & 0 & 100 \\
\hline Chloramphenicol & 8 & 80 & 20 \\
\hline Nitrofurantoin & 4 & 100 & 0 \\
\hline \multicolumn{4}{|c|}{$\begin{array}{c}\text { Table 2. Percentage of Antibiotics Prescribed to } \\
\text { Treat Postpartum Sepsis Patients }\end{array}$} \\
\hline
\end{tabular}

\section{DISCUSSION}

Postpartum sepsis continues to be a fundamentally major health issue which should be investigated thoroughly for the therapeutic need as well as for preventive and promotive well-being.

The more prominent influenced patient found in present study was at an age of 20-25 years. In the present study, most of the patients were in the age group of 20-30 years. This finding was little similar to that of the study done in Comilla Medical College Hospital, it was found 59\% cases in the same age group. The highest incidence of puerperal sepsis in younger age group of females might be due to the reason that majority of women get pregnant in this age group. The age distribution observed in the present study was also consistent with previous study done in the same department of Mymen Singh Medical College Hospital and in Dhaka Medical College Hospital ${ }^{10}$. In the present study the highest mode of delivery was found to be through caesarean technique. The reason may be risk to the mother and infant, high blood pressure, breech position or issue with the umbilical cord.

The examination of the data on the aetiology of postpartum sepsis in the present study in which $28 \%$ of the respondents shows that by Gram positive Enterococcus species, $20 \%$ signifies with Klebsiella species. The study showed that common bacteria that invaded in the participants were Enterococcus species, followed by Klebsiella species. However, the present investigation found in contradictory with the findings of UK population-based case control study states that the common bacteria were E. coli and Group A Streptococcus. A similar study was conducted in which the report shows that the most common pathogen isolated was Escherichia coli. ${ }^{11}$ The organisms isolated from the maternal genital tract and baby's surface colonization were correlated with those isolated from blood culture by calculating Phi correlation coefficient. The result found to be was $E$. coli as the most common organism isolated from maternal genital tract and surface cultures of babies. ${ }^{11}$ The data isolated in the present study indicated Enterococcus as the predominant bacteria that eventually lead to sepsis.

An elevated TLC level was observed among the sepsis patients. This is perhaps owing to infection. Temperature of 
the different patients during hospital period was found to be elevated. Pyrexia increases because it is one of the symptoms of postpartum sepsis stated by WHO. The present study demonstrated the antibiotic sensitivity and resistance of the isolated bacteria. The highest sensitivity of drug was found to be streptomycin, vancomycin, linezolid, nitrofurantoin and fosfomycin in decreasing order and highest resistant was levofloxacin, cefotaxime, penicillin, norfloxacin, ciprofloxacin, ceftriaxone, erythromycin, cefepime and cephalothin.

The susceptibility of antibiotics to individual organism has been depicted. All the strains of Klebsiella species were resistant to chloramphenicol, tetracycline, and tobramycin. $E$. coli strains were completely resistant to tetracycline, tobramycin, azithromycin, piperacillin-tazobactam and chloramphenicol. Staphylococcus aureus were resistant to vancomycin and linezolid. Staphylococcus epidermidis were found to be resistant to amoxicillin-clavulanic acid, piperacillin-tazobactam, vancomycin, ofloxacin and linezolid. Enterococcus species were resistant to streptomycin, vancomycin, linezolid, nitrofurantoin and fosfomycin and for mixed growth chloramphenicol, vancomycin and linezolid were found to be resistant.

In the present study the most commonly prescribed antibiotics was metronidazole and gentamicin. These antibiotics are the first line treatment for postpartum sepsis thus health personnel are adhered to the current guidelines.

\section{CONCLUSIONS}

Enterococcus and Klebsiella species are the common bacteria isolated in postpartum sepsis. Enterococcus species was found to be highly susceptible to streptomycin, vancomycin and linezolid and Klebsiella species to tetracycline, tobramycin and chloramphenicol. Drugs to which organisms were found to be resistant in the study were levofloxacin, penicillin, norfloxacin, ciprofloxacin, ceftriaxone, erythromycin, cefepime and cephalothin. Thus, these drugs may not be prescribed in the empirical therapy of postpartum sepsis. To counteract the burden of puerperal infections on neonatal mortality, minimising infection in the peri-partum period using appropriate measures and antibiotics prophylaxis on one side, and reducing the risk of maternal infection through antenatal care and nutritional supplementation are needed.

\section{REFERENCES}

[1] Momoh MA, Ezurgwuwie OJ, Ezeigwe HO. Causes and management of puerperal sepsis: the health personnel view point. Advanced Bio Research 2010;4(3):154-8.

[2] Dole C, Stein C. Global burden of maternal sepsis in the year 2000: evidence and information for policy (EIP), World Health Organization, Geneva, 2003.

[3] Special bulletin on Maternal Mortality in India 20102012. Sample Registration System Office of Registrar General, New Delhi, India.

[4] Special bulletin on Maternal Mortality in India 2004-06. Sample Registration System Office of Registrar General, New Delhi, India.

[5] Jayanthi G. A study to assess the knowledge and attitude on prevention of puerperal infection among staff nurses at selected hospital Bangalore. 2007.

[6] Patra S, Singh B, Reddaiah VP. Maternal morbidity during postpartum period in a village of north India: a prospective study. Trop Doct 2008;38(4):204-8.

[7] Bang RA, Bang AT, Reddy MH, et al. Maternal morbidity during labour and the puerperium in rural homes and the need for medical attention: a prospective observational study in Gadchiroli, India. BJOG 2004;111(3):231-8.

[8] Maharaj D. Puerperal pyrexia: a review. Part I. Obstet Gynecol Surv 2007;62(6):393-9.

[9] Acosta CD, Knight M. Sepsis and maternal mortality. Curr Opin Obstet Gynecol 2013;25(2):109-16.

[10] Education material for teachers of midwifery. Midwifery education modules - second edition. Managing puerperal sepsis. World Health Education (WHO) 2008: p. 64.

[11] Kerur BM, Bhat VB, Harish BN, et al. Maternal genital bacteria and surface colonization in early neonatal sepsis. Indian J Pediatric 2006;73(1):29-32.

[12] Acosta CD, Kurinczuk JJ, Lucas DN, et al. Severe maternal sepsis in the UK, 2011-2012: a national case-control study. PLoS Med 2014;11(7):p. e1001672. 